\title{
Déconstruction-reconstruction identitaire et poétique de l'altérité dans Le procès-verbal de J-M G. Le Clézio
}

\section{Abdoulaye DIOUF}

À partir de la fiction narrative, Jean-Marie Gustave Le Clézio pose de façon originale la réflexion philosophique sur l'identité et l'altérité dans son premier roman Le procès-verbal (1963). La marginalité et l'individuation d'Adam Pollo, la symbolique de son nom qui renvoie en partie au premier homme qu'il cherche à redevenir à travers la postulation de la nudité, la métamorphose que le personnage subit ainsi que la palinodie qu'il réalise par la suite en s'ouvrant à la communauté des hommes (donc à l'Autre) sont autant d'éléments, parmi tant d'autres, qui l'attestent. Bien plus, ces éléments prouvent, à travers le dynamisme qui les caractérise pour la plupart, que l'identité n'est pas une donnée figée chez Le Clézio. Elle procède d'un processus qui combine deux mouvements rhizomatiques qui ne sont contradictoires qu'en apparence: déconstruire pour mieux reconstruire. Dans le second mouvement de la reconstruction, l'identité se trouve structurée par le rapport au monde et par la présence de l'Autre, malgré « la faille identitaire» (Poulet, n.p.), « le leurre identitaire » (Labbé 106) et « la mise en solitude du personnage » (Amar 77) soulignés par la critique qui s'intéresse à son œuvre et dont j'entends prolonger la réflexion dans cet article. C'est pour dire que la réclusion du personnage le clézien n'empêche pas son rapport au monde à partir duquel il reconstitue «une identité ». Et ce qu'il ne faudrait pas comprendre par cette marginalisation du personnage, c'est que Le Clézio veuille lancer Adam Pollo dans la quête d'une nouvelle identité qui lui serait propre.

Le présent article formule l'hypothèse que Le Clézio, à travers son personnage principal, marque une étape vers l'altérité dans le sens de tout ce qui est autre, étape que les alchimistes appellent « l'œuvre au noir » et qui coïncide avec la phase de dissolution, de calcination et de séparation des oripeaux identitaires. À partir de ce moment, et comme le souligne l'ethnoanthropologue français Marc Augé, " c'est toujours la réflexion sur l'altérité qui [...] permet toute définition identitaire » (84). Comment, sur les ruines de l'identité dite immuable, Le Clézio reconstruit-il une autre identité en rapport avec l'altérité? Quelles sont les stratégies de mise en altérité qui participent de l'entreprise de gommage de cette conception de l'identité dans le 
roman ?

\section{De la migration de l'identité chez Le Clézio}

Dans Le procès-verbal, roman séminal où Le Clézio inaugure sa conception de l'identité que la suite de son œuvre va poursuivre, la question, dans sa complexité plastique, y apparaît sous les semblances d' une invalidation de toute individuation poussée, d'une disqualification de toute postulation singulière et d'une récusation de toute revendication essentialiste dans la tentative de saisie de soi. Cette dernière entreprise passe, selon Le Clézio, par la médiation d'une subjectivité autre et d' un rapport au monde. De ce point de vue, sa conception de l'identité, même si elle n'évacue pas littéralement la question du sujet, ne s'accommode pas de la clôture de cette conscience de soi comme sujet précédemment développée dans les philosophies « égologiques » par Descartes (cogito ergo sum), Kant (Ich Denke), Husserl (le « Je-tout puissant »). On sait, par exemple, que le «Je pense donc je suis » cartésien, dans le sillage du moi monadique de la culture occidentale, repose sur l'idée que la coïncidence avec soi-même se fait indépendamment de toute médiation, que la connaissance immédiate et intuitive de son être passe par la seule pensée réfléchie.

Dans Le procès-verbal, Le Clézio procède par une démarche dialectique qui met d'abord en scène un personnage, Adam Pollo, à qui il prête un projet anthropologique (redevenir le premier homme) et une quête philosophique (s'affranchir de toutes les formes de déterminisme pour être libre) qui s'avèrent par la suite illusoires. En effet, le personnage principal du roman a préféré quitter la demeure familiale pour aller vivre seul dans l'isolement d'une maison abandonnée qui, en renvoyant à la notion de «tiers espace » (Thirdspace) d'Edward Soja, recoupe ce territoire en émergence que Gilles Deleuze et Félix Guattari décrivent comme la manifestation spatiale de la transgressivité. Ainsi, Adam Pollo reflète la posture de mal-être social de l'Homme écarté de la fin du vingtième siècle rendu doublement solitaire par l'écrasement de la société moderne et la rupture avec le monde naturel. Pour échapper à cette tenaille, il trouve d'abord la planche de salut dans la postulation philosophique de la nudité qui est assimilable à une quête alchimique dans le roman. En tant que nostalgie de la transparence originelle, cette nudité montre, dès le seuil du roman, le renoncement à soi d'Adam Pollo et son aspiration à une identité, mais celle-là universelle, dépouillée de tout particularisme, qui épelle la ruine de toute forme d'appartenance fondamentale. Voilà comment s'énonce la première 
occurrence de ce désir de transparence identitaire au moyen de la nudité, devenu finalement un véritable topos dans le roman : "Il y avait une petite fois, pendant la canicule, un type qui était assis devant une fenêtre; c'était un garçon démesuré, un peu voûté, et il s'appelait Adam ; Adam Pollo. [...] Il était allongé dans une chaise longue devant la fenêtre ouverte, torse nu, tête nue, pieds nus, dans la diagonale du ciel » $(P V 15)$.

Adam « voudrai[t] bien vivre tout nu et tout noir » (213), il est à la recherche de « la femme nue » et il achète une carte postale représentant une «femme demi-nue » en bikini sur la plage (167). En voulant ainsi redevenir le premier homme, c'est-à-dire l'Adam mythique, il cherche à être transparent et à se débarrasser de son ego pour retrouver une identité en étroite relation avec une altérité dans laquelle toutes les croyances religieuses - les religions révélées tout comme celles païennes - peuvent se reconnaître de sorte à faire de lui un personnage anonyme. Il cherche ainsi, à travers le pouvoir de la re-nomination, à échapper à la logique d'appartenance à une lignée singulière surtout quand on sait que « le nom identifie l'individu en le rattachant à une filiation » (Augé 61). C'est ce que connote la double symbolique de son nom qui tient à la fois du prophétisme hébraïque (Adam renvoie au premier homme suivant le mythe judéo-chrétien) et du prophétisme grec (la contraction d'Adam et de Pollo donne Apollon, dieu du soleil, de la divination et des arts selon le mythe païen). Cette première filiation symbolique se trouve renforcée par l'identification d'Adam à Giézi ${ }^{1}$, ce serviteur d'Élisée dont on raconte l'anecdote dans Le Livre II des Rois de la Bible (chapitre 5, versets 1-27). De ce point de vue, l'identité devient transcendante chez Adam Pollo dans la mesure où elle finit par recouper l'altérité en ces termes : " nous sommes tous pareils, tous frères, hein. [...] Nous avons les mêmes corps et les mêmes esprits. C'est pour ça que nous sommes frères » ( $P V$ 243). Avec ce mouvement d'identification qui neutralise toute velléité de particularisme, le héros du Procèsverbal peut faire sien le constat de vide ontologique que fait l'auteur Le Clézio dans L'Extase matérielle comme résultat négatif de toute tentative d'exploration des profondeurs abyssales de l'être : «Faut-il fouiller au fond de soi, au plus profond, défaire, vomir tout son passé? Est-ce seulement possible? Et si on pouvait le faire, que trouverait-on qu'on ne sache déjà? En vérité, et cela, c'est la dure réalité qu'il faut dire une bonne fois pour toutes, nous ne sommes rien. Rien n'est nous, rien n'est à nous » (39-40).

C'est cette absence de l'existence d'un fond ontologique prétabli comme possession ou propriété dont on peut s'enorgueillir qui fait certainement que, chez Le Clézio, l'accès à la 
conscience subjective est à chercher nécessairement dans une démarche indirecte qui passe par le détour de l'autre : «La conscience de soi est sans doute avant tout conscience des autres. Ce n'est qu'à partir de l'instant où l'autre est ressenti comme semblable à soi, donc susceptible de jugements, de passions, de sensations, que l'homme peut, à travers le lien du langage, dégager son sentiment de vie » (92).

C'est ce que Le Clézio appelle « la gémellité de l'être » qui suggère que l'individu est tributaire d'une existence double : «être, c'est être soi par l'autre » (94). Il y a donc une dimension sociale de l'identité que Rimbaud avait traduite auparavant dans la création littéraire par la célèbre formule " Je est un autre ». Paul Ricœur l'a fait ressortir par la suite dans le domaine philosophique en remplaçant le « je » cartésien par le «Soi » comme entité beaucoup plus englobante de par son impersonnalité et sa modestie : "'Soi' est immédiatement structuré par l'altérité. [...] Dans Soi-même comme un autre, j'ai voulu montrer que l'ipséité - la propriété réflexive du soi - était essentiellement liée à sa capacité réceptive à l'égard de l'altérité » (Ethique, 24-26).

À partir de ce moment, l'isolement d'Adam Pollo, par lequel il s'éloigne de la causalité rationnelle des lois d'une société qu'il juge absurde, est à lire autrement comme un principe positif. Il procède d'une démarche à travers laquelle il cherche à renégocier son rapport au monde selon sa guise. Pour cela, il doit d'abord se tenir à distance du conventionnalisme familial et de ses ramifications identitaires auxquels le convie sa mère Denise Pollo dans la lettre qu'elle lui adresse : «En dépit de ce que tu peux paraître, nous resterons toujours le clan des Pollo. Tu portes le nom et le prénom d'un de nos ancêtres. L'arrière-grand-père s'appelait Antoine-Adam Pollo - tu dois être une part importante de ce clan, même si tu ne fais pas comme les autres même si tu te singularises par ailleurs » ( $P V$ 239-240).

Ainsi s'opère, par le biais de l'oubli fondateur de toute quête de liberté, la première brisure d'un support important de l'identité - la famille - dont les ramifications cherchent à rattacher Adam à une ascendance («ses ancêtres ») qu'il ne voudrait pas revendiquer pour éviter de porter atteinte à ses convenances et exigences personnelles. Ce que le héros du Procès-verbal réalise à travers son attitude, c'est une véritable mise en question du modèle de l'ascendance et de la descendance arborescentes que Pierre Rosenstiehl et Jean Petiot appellent « l'imagerie des arborescences de commandement ${ }^{2} »$ (Deleuze et Guattari 25) et qui renvoie, selon Deleuze et Guattari, à des systèmes centrés ou des structures hiérarchiques desquels relève l'institution de la 
famille.

Dans cet énoncé de Denise Pollo, l'identité apparaît comme permanence à travers l'emploi de l'adverbe «toujours » qui renvoie à ce «maintien de repères fixes, constants, échappant aux changements pouvant affecter le sujet ou l'objet par le cours du temps »dont parle André Green ${ }^{3}$. Adam Pollo lui oppose une sorte de mort symbolique à la famille des hommes (déterritorialisation ${ }^{4}$ avec le renoncement à son identité sociale) pour une renaissance dans le règne du sacré (reterritorialisation dans la maison abandonnée qu'il squatte, où il fait le sacrifice rituel du rat et qui devient un Temple sacré). Le Fils de l'homme meurt pour laisser vivre celui de Dieu. C'est d'ailleurs pourquoi Ook Chung parle de «baptême prophétique d'Adam » (68) suivant l'idée d'une conception dynamique de l'identité comme mouvement perpétuel de re-naissance que Claudio Magris exprime de la manière suivante :

L'identité n'est pas une donnée rigide et immuable, elle est fluide, c'est un processus toujours en devenir, par lequel on s'éloigne continuellement de ses origines, comme le fils quitte la maison de ses parents et on y retourne par la pensée et le sentiment; c'est quelque chose qui se perd et qui se renouvelle, dans un mouvement incessant de dépaysement et de retour... Une patrie et une identité ne peuvent pas se posséder comme on possède une propriété. (92)

Dans Le procès-verbal, l'identité ne s'offre pas comme un substrat immuable: elle subit des fluctuations multiformes. En atteste la métamorphose d'Adam Pollo en rat qui montre surtout comment le personnage le clézien construit son altérité en rapport avec cet être animal avec lequel il présente une gémellité : ils sont tous les deux squatters. Cette métamorphose, en tant que déviation de la trajectoire de son identité essentialiste pour l'embrayage sur la voie de l'insaisissable et de la non-identité, constitue l'étape suprême de renoncement à une partie de son identité humaine : «Adam se transformait en rat blanc, mais d'une métamorphose bizarre : il gardait toujours son corps à lui, ses extrémités ne devenaient pas roses, et ses dents de devant ne s'allongeaient pas; non, ses doigts sentaient toujours le tabac, ses aisselles la sueur, et son dos restait plié en avant, dans la position accroupie, tout près du plancher, conditionné par la double cambrure de la colonne vertébrale » (118).

À ce stade de l'évolution de son personnage, Le Clézio transcende la notion d'espèce et opère, avec la métamorphose, une vaste entreprise de gommage des identités qu'Élisabeth Poulet analyse dans le sillage de la fluidité en termes de « déplacement », de « déroulement fuyant » qui déjoue la fixité d'un discours pour apparaître finalement comme « le signe d'une non-identité » 
(n.p.). En dehors du rat avec lequel il a « un air de parenté » ( $P V$ 117), Adam développe également une relation quasi fusionnelle avec le chien, son ami et compagnon à l'époque de l'isolement, derrière lequel il court pour vivre sa solitude et passer indifférent devant la race humaine. Désormais, « il pouvait aller et venir dans les rues de la ville, fouiner dans les magasins » et « bientôt peut-être, il pourrait lui aussi uriner tranquille sur les essieux des voitures américaines ou les panneaux d'interdiction de stationner, et faire l'amour en plein air, en pleine poussière, entre deux platanes » (110). Chevauchement d'espèces, mais chevauchement de genres également qui joue sur la confusion pour obvier à la logique de classification binaire : « Une femme-adam, prise dans sa robe pourpre, court derrière l'homme-adam, en claquant fort ses talons aiguille $\gg(185)$.

En reprenant les distinctions que Deleuze et Guattari établissent entre « l'arbre » et « le rhizome » pour signifier respectivement la « filiation » et l'« alliance » (36), on se rend compte que, in fine, l'identité postulée par le personnage le clézien s'apparente métaphoriquement plus à l'« identité-rhizome » qu'à l'« identité-racine ». Le rhizome étant cette tige souterraine qui se singularise par ses multiples embranchements et ramifications synonymes de connexion, de multiplicité et d'hétérogénéité :

[...] à la différence des arbres ou de leurs racines, le rhizome connecte un point quelconque avec un autre point quelconque, et chacun de ses traits ne renvoie pas nécessairement à des traits de même nature, il met en jeu des régimes de signes très différents et même des états de non-signes. [...], le rhizome est un système acentré, non hiérarchique et non signifiant, sans Général, sans mémoire organisatrice ou automate central, uniquement défini par une circulation d'états. $(31-32)$

Cette « identité-racine », avec sa structure arborescente figée, montre que la question de l'identité a partie liée dans le roman le clézien avec celle de la liberté. Et c'est sans doute la raison pour laquelle Adam s'intéresse aux animaux qui sont tout à fait libres de se livrer, en tout lieu, à la satisfaction de leurs appétences primitives. Si Le Clézio récuse cette notion d'« identitéracine ", c'est pour mieux situer son personnage sur le chemin de la contestation de toute cette part déterministe de l'Homme qui ne dépend pas de lui et qui a trait à des liens identitaires fondamentaux comme le sang (la famille), la race, l'espèce ou le genre. À vrai dire, ce n'est que dans la liberté qu'on peut appréhender la différence constitutive de l'altérité à partir de laquelle on reconnaît son identité. C'est ce que pense Raymond Mbassi Atéba lorsqu'il affirme que « la poétique de l'identité et de la fluidité chez Le Clézio transite par la poétique de la liberté et de la 
mondialité : seul l'homme libre peut vivre en harmonie avec son identité » (340). Avec l'implication consciente du sujet que tout cela suppose, indépendamment de la prise en compte de toute antériorité, la conception de l'identité chez Le Clézio s'abreuve à la source de la démarche constructiviste dans une perspective qui finit par faire litière de la clôture de soi pour mieux s'ouvrir à l'Autre, bref à tous les êtres.

Nous appelons cette «identité-rhizome » identité transcatégorielle chez Le Clézio parce qu'elle favorise la culture de l'enjambement qui lui permet de conjoindre à la fois l'humain, l'animal, le végétal et même le rupestre dans une sorte de fluidité symbiotique soutenue par une certaine vision du monde. Cela implique un mouvement qui montre ainsi comment la conception de l'identité, chez lui, tient en partie de ce que Ricoeur appelle, dans son anthropologie philosophique développée dans Soi-même comme un autre, l'ipséité (identité variable) par opposition à la mêmeté (identité immuable) :

Je suis frappé par le fait que, sous le mot «identique » ou «identité », nous mettons deux significations tout à fait différentes: selon la première, l'identité c'est ce qui ne change pas; c'est l'immuable. Il s'est fait une sorte de fusion avec les philosophies du sujet en «je» (première personne) et de l'identique (en immutabilité), comme s'il y avait un substrat qui ne bougeait pas derrière les changements. C'est cette collusion justement entre l'identité immuable et la première personne toute-puissante que j'ai essayé de briser en donnant un autre sens à «identique», qui est cette sorte d'identité que nous connaissons par la fidélité à la parole donnée; ici, «identique » ne veut pas dire que je ne change pas, mais que malgré mes changements, je me maintiens dans une obligation. (227)

En bref, la problématique de l'identité dans Le procès-verbal ne se réduit pas à la recherche d'une certaine idiosyncrasie que semble suggérer la retraite d'Adam Pollo. Il faut plutôt lire sa rupture de ban comme un refus de toute forme de conventionnalisme, gage de la liberté à partir de laquelle il entrevoit un nouveau rapport au monde qui lui permet de reconstruire une identité captive du fronton de tous les êtres. Mais pour y arriver, le roman met en scène des stratégies qu'il convient d'analyser à présent.

\section{La mise en altérité dans le roman}

Dans l'analyse qu'elle fait de l'altérité sous l'angle de ses formes et de ses figures, Denise Jodelet la conçoit comme un processus graduel avec deux pôles qui s'étendent d'une part de la « reconnaissance d'une proximité et d'une similitude au positionnement dans une extériorité 
radicale », et d'autre part « de l'interdépendance ou l'intersubjectivité à l'étrangeté absolue » (23-24).

Dans Le procès-verbal, Adam Pollo renverse littéralement l'ordre des termes du premier pôle et les rapproche en infléchissant sa position d'extériorité pour adopter par la suite les postures de fusion, d'identification et de simultanéité, symptomatiques d'une altérité dont il éprouve fortement le besoin, car à l'interpellation de Julienne « vous en avez assez d'être seul », il répond « oui, c'est ça. Je voudrais être avec les gens » (295). L'altérité, en tant que médiatrice de l'identité, acquiert ainsi une fonction ontologique chez Adam Pollo en ce sens qu'elle lui permet de combler le vide de son existence, d'après ce qu'il confie à l'étudiant aux lunettes : « C'est comme cette discussion. Elle ne m'intéresse pas pour ce qu'elle est, pour ce qu'elle a l'air d'être. Mais seulement dans la mesure où elle remplit un vide. Un vide terrible, insoutenable. Entre les deux niveaux de la vie ... Entre deux paliers, deux temps, vous comprenez ? » (297).

Tout cela atteste encore, tel que le montre Michel Foucault dans l'analyse généalogique de la notion de sujet qu'il propose dans la lignée de la pensée occidentale de l'altérité (autour de Freud, Lacan, Althusser, Levinas, Heidegger, Derrida, Sartre, etc.), la complexité de l'identité qui participe à la fois d'une identité de soi à soi (fondement du cogito cartésien) et d'une identification à la norme (fondement de la morale kantienne et de son impératif catégorique).

S'il apparaît dans le postulat de cet article que l'isolement d'Adam n'est pas aussi exempt d'altérité que son incommunicabilité pourrait le laisser penser, c'est parce que c'est une étape que le personnage met à profit pour entrer en communion avec le monde naturel à la suite de sa rupture avec la ville caractérisée par la «surmodernité $e^{5}$. À la faveur de cette solitude, Adam Pollo s'essaie à ce qu'il appelle « la voie des certitudes qui est celle de l'extase matérialiste » ( $P V$ 204) qui le plonge dans l'éternité où « règne la matière intemporelle » et où « il n'y aura plus guère qu'un homme, plus guère qu'une femme " (184). Moment où l'âme s'échappe de la clôture et de l'emprise du corps, l'expérience de l'extase et de la contemplation permet à Adam de renoncer à soi pour une symbiose organique avec les autres êtres naturels. Voici comment il rabroue Michèle pour avoir interrompu son expérience : "'Idiote!' répondit Adam, 'd'avoir interrompu ma contemplation ! [...]. Rien, rien ... Je ne peux pas t'expliquer. J'étais déjà arrivé au végétal... Aux mousses, aux lichens. C'était tout près des bactéries et des fossiles. Je ne peux pas t'expliquer' » (78). 
En tant que fusion avec le monde dans le silence, l'extase matérialiste permet à Adam de vivre tout autrement l'angoisse existentielle née du rapport mécanique de l'Homme au monde. Elle se réalise à travers le double système de la multiplication et de l'identification (donc par le biais de l'altérité) qui permet au personnage de s'anéantir dans l'oubli de son identité individuelle. Adam devient ainsi « le maître des choses » (35) avec une conscience sphérique qui le place aux confluents de toutes les réalités du monde, violenté « non plus par des milliards de mondes, mais par un monde seul et unique [où] il avait «fait la jonction de tous les temps et de tous les espaces » (92) :

Non, ce qui agit Adam, c'est la réflexion, la méditation lucide. Partant de sa propre chair humaine, de sa somme de sensations présentes, il s'anéantit par le double système de la multiplication et de l'identification. [...] Bientôt il n'existe plus. Il n'est plus lui-même. Il est perdu, faible parcelle qui continue à se mouvoir, qui continue à se décrire. Il n'est plus qu'un vague revenant, seul, éternel, démesuré, terreur des vieilles femmes solitaires, qui se crée, meurt, vit et revit et s'engloutit dans l'obscur, des centaines, des millions et des milliards d'une fois infinie, ni l'un ni l'autre. (205)

Dans ces moments de communion poussée avec les éléments du monde animal, végétal et rupestre lors desquels Adam Pollo cultive une autre forme d'altérité, le personnage se donne à voir comme le double de son auteur particulièrement fasciné par ce mode de vie caractéristique de la société primitive des Amérindiens où le je ne s'oppose pas à l'Autre et dont il vante le modèle pour mieux déconstruire la subjectivité monadique de la culture occidentale. Pour ce peuple, l'homme est lié aux choses et au cosmos dont il est une composante (unité que recherche Adam Pollo). C'est dire que, pour être né en France, avoir vécu à l'île Maurice, séjourné chez les Indiens Emberas et Waunanas et dans d'autres parties du monde, Le Clézio s'enfermerait mal dans la clôture d'une seule identité. C'est pourquoi, dans son discours à l'Académie suédoise lui décernant le prix Nobel, il célèbre une œuvre placée sous le signe de la rupture - la brisure du nom - en affirmant le refus d'être assigné à la signification de «Le Clézio » qui renvoie à «enclos », « clôture » en breton. L'étiologie de ce malaise permet de voir que l'auteur de Le procès-verbal est résolument un homme d'ouverture. Son parcours, qui se trouve à la croisée de trajectoires multiples, aux fluctuations diverses, à la manière d'une fractale, est sans doute source de sa crise d'identité originelle dont a parlé la critique de son œuvre et qui explique le sens des Autres qui l'habite et son idéalisation permanente de l'altérité. Au sujet de cette nostalgie du primitif, Le Clézio lui-même confie ce qui suit à Jean-Louis Ézine : 
Si j'idéalise la société amérindienne, c'est qu'elle n'avait pas fait de la propriété son principe de base. Les sociétés amérindiennes considéraient la possession de la terre comme une hérésie, comme quelque chose d'absurde. Les sociétés amérindiennes qui survivent considèrent toujours que la terre est à tout le monde. C'est notre mère à tous, pour le profit de chacun, et personne n'a le droit de dire que la terre lui appartient. Elle lui est prêtée, comme la vie d'ailleurs, comme les éléments, comme l'air. (58-59)

Finalement, à travers ce contact avec d'autres réalités, il se produit une altération de l'identité singulière d'Adam. À la jointure de plusieurs appartenances identitaires, le personnage devient une figure traversière à l'identité composite. Son être (polyphonique ?) peut entonner plusieurs chansons et répondre d'une porosité à tous les souffles.

Mais c'est fondamentalement l'identification, autre étape de la mise en altérité du personnage, qui est à la base de cette expérience de la fusion ou de l'extase matérialiste. Elle repose chez Le Clézio sur l'idée que l'être ne s'arrête pas à l'humain. Dans son appareil de pensée, l'identification coïncide avec ce qu'il appelle « l'assimilation » comme étant le premier mouvement vers la conscience de soi (je suis homme) avant l'étape de la «différenciation » (je ne suis pas n'importe quel homme) (Extase, 92). Dans Le procès-verbal, elle s'illustre lors de la scène de noyade d'un homme de quarante ans à laquelle Adam Pollo assiste. Ici, le spectateur qui était détaché commence à s'humaniser en exprimant le pathos d'un ressentir commun qui lui permet d'accepter son identité à partir de l'altérité des autres, condamné comme tout le monde entre la vie et la mort: « et tous, on est le cœur serré par un passage étrange; le corps de l'homme mort, maintenant, se dépouillait tranquillement de son souvenir risible » $(154$; je souligne). Il s'adresse désormais à tous les hommes confondus dans la même identité, celle de l'Homme tout court. Sorti de son mutisme et de sa solitude, il demande aux gens d'apprendre à parler pour montrer toute son envie de quitter la clôture de l'incommunicabilité avec les Hommes où le confinait sa propre marginalité : «Apprenez à parler. Essayez, vous aussi. Même si vous n'avez rien à dire. Puisque je vous dis qu'on vous donne la parole. Pourquoi ne pas essayer, tant que vous êtes, de remplacer vos propres machines : allez, parlez, de droite et de gauche. Propagez la bonne parole » (246-247).

Avec ce nouveau don de la parole « aux obscurités mystérieuses » (248), Le Clézio réalise la parodie de la révélation prophétique. Comme Moïse au sommet de la colline, Adam reçoit une illumination à la faveur d'une révélation surnaturelle ${ }^{6}$ qui lui permet d'adopter « un ton, entre le prêche fanatique et la harangue de repas de noces » (246-247). Avec cette nouvelle 
figure de prophète qu'il incarne - figure d'union et d'altérité -, Adam développe un nouvel anthropocentrisme en descendant parmi les hommes avec un langage purifié : «Tout à coup, sur terre, tout fut changé. Oui, d'un seul coup, je compris tout. Je compris que cette terre était mienne, et à nulle autre espèce vivante. Pas aux chiens, pas aux rats, pas à la vermine ni à rien. Pas aux escargots, ni aux blattes, ni aux herbes, ni aux poissons. Elle était aux hommes. Et puisque j'étais un homme, à moi » (248-249).

Redescendu dans la société des Hommes, donc reterritorialisé, la quête de la transparence originelle étant utopique, Adam Pollo va tenter de retrouver l'unité de son être par le moyen de la simultanéité qui, en tant que recherche de l'harmonie, apparait en ces termes comme une confrontation de son expérience avec diverses autres expériences :

La simultanéité est un des éléments nécessaires à l'Unité qu'Adam avait un jour pressentie, soit au cours de l'histoire du zoo, soit à cause du Noyé, soit à propos de bien d'autres anecdotes qui sont volontairement oubliées ici. La simultanéité est l'anéantissement total du temps et non du mouvement [...] Il s'agit, à propos d'un acte quelconque, mettons, fumer une cigarette, de ressentir indéfiniment durant le même geste, les millions d'autres cigarettes vraisemblablement fumées par des millions d'autres individus sur la terre. (203)

Mais puisque son expérience diffère de celle des autres, on voit comment cette quête de l'harmonie devient une tentative de conciliation des contraires. Et c'est là qu'on trouve la question philosophique centrale que pose le roman de Le Clézio et qui lui valut, selon la critique, la distinction du prix Nobel en 2008: comment penser le pluriel et la différence sans rompre l'unité?

\section{Conclusion}

Chez Le Clézio, la conception de l'identité dépasse la simple question de l'appartenance sociale et des traits fondamentaux par lesquels on peut manifester une individualité propre. Et cette identité d'un individu, aussi individuelle qu'elle soit, finit par être fuyante, c'est-à-dire inscrite sur une ligne de fuite à la faveur du double mouvement de la « déterritorialisation » et de la « reterritorialisation ». C'est ce qui explique l'itinéraire rhizomatique qu'emprunte Adam Pollo, fait de renoncements qui le mènent de plus en plus loin de sa famille, de son espèce et de son genre par le moyen d'un dépouillement à la progression graduelle (marginalité, nudité, métamorphose). Elle recoupe, dans le principe, les réflexions philosophiques sur l'altérité qui évacue toute modalité directe et immédiate de saisie de soi. Mais, en favorisant une communion 
poussée de son personnage avec le monde naturel jusqu'à la transvaluation, en le faisant redescendre chez les Hommes avec un langage nouveau et purifié chargé de porter la bonne parole à une société dont il avait dénoncé la comédie factice, Le Clézio élargit le champ de l'altérité philosophique. Pour cela, il confère à l'Autre une identité composite qui dépasse l'intersubjectivité (dans le sens du rapport d'un sujet avec un autre sujet) pour embrasser les choses dans un rapport au monde plus global que le personnage principal réalise en adoptant plusieurs postures dans le roman (fusion, identification, simultanéité).

D'une poétique de l'identité qui transite par l'altérité au moyen du mouvement de la déconstruction-reconstruction, on aboutit finalement à une poétique des valeurs dans le premier roman de Le Clézio à travers laquelle il propose un modèle renouvelé de la vision des choses qui tendrait vers une forme d'humanisme où l'on cultive à la fois « l'ambiguité ${ }^{7}$ » de l'altérité (qui ne réduit jamais intégralement la personne ni à elle-même ni à autrui) et «l'ambivalence » de l'identité (qui peut cumuler plusieurs appartenances). En s'érigeant ainsi contre les systèmes centrés et les structures hiérarchiques, en développant une défaillance mémorielle, Adam Pollo fait véritablement rhizome, dans le sens de Deleuze et Guattari, en se présentant comme un personnage "antigénéalogique », sans identité fixe, qui procède par variation, expansion, conquête.

\section{Bibliographie}

Amar, Ruth. Les structures de la solitude dans l'œuvre de J.M.G. Le Clézio. Paris : Publisud, 2004.

Atéba, Raymond Mbassi. Identité et fluidité dans l'œuvre de Jean-Marie Gustave Le Clézio. Une poétique de la mondialité. Paris : Harmattan, 2008.

Augé, Marc. Le sens des autres. Actualité de l'anthropologie. Paris : Fayard, 1994.

Chung, Ook. Le Clézio. Une écriture prophétique. Paris : Imago, 2001.

Deleuze, Gilles et Félix Guattari. «Introduction : rhizome ». Mille plateaux. Capitalisme et schizophrénie. Paris : Minuit, 1980.

Ézine, Jean-Louis. Ailleurs ; entretiens sur France-culture. Paris : Arlea, 1995.

Jodelet, Denise. «Formes et figures de l'altérité ». L'autre: regards psychosociaux. Dir. Margarita Sanchez-Mazas, Margarita et Laurent Licata. Grenoble: PU de Grenoble, 
2005. 23-47.

Labbé, M. Le Clézio. L’écart romanesque. Paris : Harmattan, 1999.

Le Clézio, J-M.G. Le procès-verbal. Paris : Gallimard, 1963.

---. L'extase matérielle. Paris : Gallimard, coll. Folio/essais, 1967.

Lévi-Strauss, Claude, dir. L’identité. Paris : Quadrige/PUF, 1995 (1977).

Magris, Claudio. Utopie et désenchantement. Paris : Gallimard, coll. L’arpenteur, 2001.

Poulet, Élisabeth. «La faille identitaire chez les personnages lecléziens ». http://www.larevuedesressources.org/la-faille-identitaire-chez-les-personnages-de-leclezio,801.html, consulté le 14 septembre 2014.

Ricoeur, Paul. Soi-même comme un autre. Paris : Seuil, 1991.

---. Éthique et responsabilité. Genève : La Baconnière, 1994.

\section{NOTES}

${ }^{1}$ Il fut atteint de lèpre par la punition de Dieu pour avoir confisqué le présent que Naaman a offert à son maitre Élisée en signe de reconnaissance.

${ }^{2}$ Dans « Automate asocial et systèmes acentrés », Communication 22, 1974, cité par Deleuze et Guattari dans «Rhizome », Mille plateaux, 1980.

${ }^{3}$ Il répertorie aussi d'autres caractères de l'identité à côté de la permanence : unité et reconnaissance du même. Voir son article «Atomes de parenté et relations œdipiennes », L'identité, dir. Claude Lévi-Strauss, 81-82.

${ }^{4}$ Deleuze et Guattari emploient les termes de déterritorialisation et reterritorialisation comme double mouvement de rupture avec le fondement-racine caractéristique de la pensée occidentale (sur le modèle de l'arbre et de la descendance) à partir duquel il définit le rhizome comme une antigénéalogie. "Rhizome », Mille plateaux, 18.

${ }^{5}$ Augé propose le concept de « surmodernité » à l'opposé de la « postmodernité » pour renvoyer à trois mots d'ordre (convergence des histoires, déterritorialisation des espaces et libération des individus) qui finissent par confiner l'homme de la modernité contemporaine à un «non-lieu » où personne n'est chez soi, mais où on est jamais chez les autres. Le sens des autres, 165.

${ }^{6}$ Adam raconte lui-même que c'est par le moyen d'un bruit sourd annonçant l'apparence d'une vieille femme ridée rencontrée sur une route qu'il découvre le visage de la terre des hommes ( $P V$ 249-251).

${ }^{7}$ Augé distingue ainsi « l'ambivalence » (qui supporte des jugements à la fois contraires et pertinents : bon/méchant, vrai/faux) de «l'ambiguïté » (ni bon ni méchant, ni vrai ni faux) en les appliquant au couple identité/altérité, 61. 